\title{
Sociedades posneoliberales en América Latina y persistencia del extractivismo
}

\author{
Postneoliberal Societies in Latin America \\ and the Persistence of Extractivism
}

\author{
Jaime Ornelas Delgado*
}

\begin{abstract}
Resumen
Los gobiernos progresistas de Latinoamérica no han logrado desprenderse del extractivismo; pero de ahí no puede concluirse que nada hayan hecho para frenar la depredación provocada por el capitalismo neoliberal; por el contrario, el uso del excedente económico o de la renta petrolera por parte de esos gobiernos para mejorar las condiciones de vida de la población y transformar la estructura productiva de sus países, marca una enorme diferencia con el neoliberalismo.

El reconocimiento de este esfuerzo, no significa caer en la ilusión de creer que los gobiernos progresistas de la región han logrado modificar los patrones de producción y consumo capitalistas, la realidad es que aún dependen del extractivismo para elevar la producción y el consumo; pero sobre todo, no se puede decir que el cambio de finalidad política del extractivismo, haya resuelto los impactos sociales y ambientales provocados, por ejemplo, por la minería a cielo abierto o los monocultivos transgénicos.
\end{abstract}

Palabras clave:

- Economía política del capitalismo

- América latina y el caribe

- Medición del crecimiento económico

\begin{abstract}
Latin american progressive goverments have not been succeful in giving up extractivism. Although, it cannot be said that nothing has been made to stop the depredation $\mathrm{ca}$ rry out by neoliberal capitalism. On the contrary, the use of the economic exceeds and the oil profits to improve living standards in the population and to transform the productive infrastructure in those countries represents a real difference from neoliberal approaches. The acknowlege of this effort, does not mean to believe that progressive governments in Latin American have been successful in changing capitalism production and consumption patterns. Indeed, those governments are extractivism dependent in order to increase production and consumption. Over all, it cannot be said that the change of extractivism political orientation has solved the social and environmental consequences of, for example, open air mining or genetically modified organism.
\end{abstract}

Keywords:

- $\quad$ Political Economy of Capitalism

- Latin America; Caribbean

- Measurement of Economic Growth

JEL: P16, N36, O47

\section{Introducción}

Los nocivos efectos de la crisis se prolongan desde su inicio en el otoño de 2007, hasta este momento con impactos diferenciados en el mundo y sin tenerse a la vista salidas viables a los problemas del proceso de acumulación a nivel planetario.

La profundidad, los impactos múltiples y una prolongada duración de la crisis, entre otras cosas, han fortalecido la certeza de la capacidad de la mo-

* Investigador Nacional Nivel 2. Centro de Estudios del Desarrollo Económico y Social (CEDES) de la 
dalidad neoliberal del capitalismo ${ }^{1}$ para forjar un futuro donde se superen los ancestrales problemas sociales agravados por la propia crisis; en consecuencia, los pueblos latinoamericanos, coincidiendo con el final del siglo xx y el inicio del Xxi, han iniciado la búsqueda de opciones a las economías basadas en el mercado autorregulado y la entrega de la riqueza social al capital privado; la privatización de los bienes públicos que satisfacen necesidades universales; la apropiación y devastación de la naturaleza; así como la destrucción del sistema de seguridad social, la apertura indiscriminada comercial y financiera, la flexibilidad laboral y el deterioro de las condiciones de trabajo, a los cuales en Latinoamérica se añade un proceso de reprimarización de la actividad económica debido a la persistencia e importancia que adquiere en ellos el modelo extractivista, que termina por fortalecer la condición dependiente de esas economías y perpetúa la división internacional del trabajo que los condena a mantenerse como proveedores de materias primas y alimentos del capitalismo desarrollado.

Actualmente, en Latinoamérica la construcción de las sociedades posneoliberales se lleva a cabo por dos vías: los "desarrollos alternativos" y las "alternativas al desarrollo":

El primer caso sirve para las distintas opciones de rectificación, reparación o modificación del desarrollo contemporáneo, donde se aceptan sus bases conceptuales, tales como el crecimiento perpetuo o la apropiación de la $\mathrm{Na}$ turaleza, y la discusión se enfoca en la instrumentalización de ese proceso; en cambio, las "alternativas al desarrollo" apuntan a generar otros marcos conceptuales y otra organización económica. Es explorar otros ordenamientos sociales, económicos y políticos de lo que veníamos llamando desarrollo (Gudynas, 2011: 42).

Los países que siguen la primera vía, se mantiene en los límites del desarrollo capitalista (y de las políticas económicas diseñadas para impulsarlo), concebido como un proceso permanente, ascendente e ilimitado guiado por el Estado para incrementar la producción de mercancías, concepción consecuente con una visión que identifica el bienestar humano "con la acumulación de objetos materiales" (Lander, 2013: 28), es decir, entendiendo el bienestar como la magnitud del valor de los bienes poseídos -siempre comparada con el valor

\footnotetext{
${ }^{1}$ Siendo el formación económica-social la expresión histórica concreta de un modo de producción, la modalidad supone una diferenciación dentro de la propia formación económica-social. Dicha diferenciación se define por las peculiaridades adquiridas por la modalidad, sin que se modifiquen sus características esenciales de la formación. En consecuencia el neoliberalismo es la modalidad actual de la formación económica-social capitalista.
} 


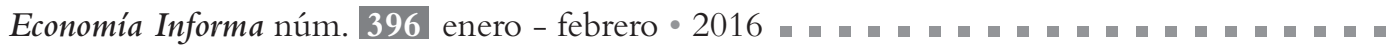

de los bienes que poseen los otros- o por la capacidad económica -mayor o menor que la de otros- para adquirir las mercancías requeridas para satisfacer sus necesidades reales o imaginadas; en cambio, la segunda vía rechaza el marco conceptual del desarrollo y explora alternativas al capitalismo donde la sociedad viva en armonía con la naturaleza, porque se reconoce parte de ella, o donde el bienestar no se mida por lo que otros carecen en comparación con lo que otros tienen y la actividad económica se oriente a satisfacer las necesidades reales de la población y deje de producirse únicamente aquello que deje ganancia.

Ambas vías tienen en común dos cuestiones: i) el esfuerzo por superar el neoliberalismo y ii) ser sociedades con gobiernos progresistas, llevados al poder político por movimientos sociales pluriclasistas que, sin renunciar a seguir siendo movimiento, decidieron participar en procesos electorales -rigurosamente controlados por el sistema procedimental de la democracia representativa-, presentando candidatos y programas de gobierno antineoliberales que lograron atraer a los votantes que dieron triunfos contundentes a las candidaturas surgidas del movimiento social.

En países como Brasil o Argentina e incluso Uruguay, se ha iniciado la construcción de sociedades posneoliberales diseñando políticas alternativas de desarrollo, es decir, sociedades que sin abandonar el capitalismo recuperan el papel regulador del Estado para llevar a cabo políticas económicas dirigidas a la modernización y el crecimiento con miras a la ampliación de la base exportadora de la economía; paralelamente, se impulsan diversas acciones orientadas a mejorar la distribución del ingreso, junto con otras políticas instrumentales -que en ocasiones devienen asistencialistas- encaminadas a reducir la pobreza, sin alterar las causas estructurales que la generan.

Este tipo de sociedades corren siempre el riesgo de terminar siendo otra modalidad del capitalismo, modalidad que por sus características se ha dado en llamar neodesarrollista, postura ya conocida en América Latina donde en sus diferentes versiones ha sostenido "que los problemas económicos y sociales que aquejaban a la formación social latinoamericana se debían a una insuficiencia de su desarrollo capitalista y que la aceleración de éste bastaría para hacerlos desaparecer" (Marini, 1977: 57). En consecuencia, el esfuerzo gubernamental se dirige a crear las condiciones generales del desarrollo capitalista y la modernización de la estructura económica, con tímidas reformas para redistribuir el ingreso y políticas poco eficaces para reducir la desigualdad social y regional.

La otra vía, se orienta a la construcción de sociedades alternativas al desarrollo, es decir, al capitalismo. Se trata de sociedades sostenidas en el creciente 
protagonismo del movimiento social y edificadas sobre un modo de producción y consumo distinto al capitalista, lo cual supone también la crítica radical al desarrollo.

Este esfuerzo no sólo ha sido práctico en el sentido de las acciones emprendidas para construir una nueva estructura productiva y de consumo, sino que también la nueva sociedad se está construyendo teóricamente, lo que ha dado lugar a la intensa discusión de categorías como el buen vivir, vivir bien, el Socialismo del Siglo XXI o innovadoras propuestas severamente críticas del capitalismo, como reconocer los derechos de la naturaleza y otras surgidas no sólo del rechazo al colonialismo y al eurocentrismo, sino también a la explotación del trabajo, la propiedad privada y a la insustentabilidad de la producción y el consumo capitalistas, el rechazo a la ideología discriminatoria y excluyente o a los valores de mercachifles burgueses y otras categorías que lo sustentan, críticas hechas en abierto rechazo a las concepciones tradicionales del desarrollo basadas en la supuesta superioridad de la cultura occidental y del capitalismo como forma de vida y de producción.

Un elemento común a las dos vías mencionadas, es el hecho de que los encuentros y desencuentros entre los protagonistas en la construcción de las sociedades posneoliberales, radican en la temática ambiental, convertida hoy en uno de los ámbitos de mayor preocupación, pues lleva implícita una nueva posición respecto de la naturaleza, a la que incluso en países, como Ecuador y Bolivia, se le reconocen derechos. Es así que hoy, cualquier discusión sobre el desarrollo o la construcción de las sociedades poscapitalistas, esté profundamente marcada por las preocupaciones ambientales y en la protección de los derechos de la naturaleza encuentren sus límites cualquier solución propuesta.

\section{Persistencia del extractivismo}

La estrecha vinculación entre la crítica al desarrollo y la temática ambiental, ha tenido como aspecto relevante el papel y peso de la actividad extractivista en la economía, actividad que no sólo comprende la minería y los hidrocarburos, pues incluye también la agricultura, la explotación forestal y la pesca orientadas a la exportación: "Sería un error, comenta Alberto Acosta, asumir que el extractivismo existe solo cuando se extraen recursos naturales o hidrocarburíferos. Hay muchas experiencias de prácticas igualmente extractivistas en la explotación de madera o en la agricultura de monocultivo" (Acosta, 2011: 85, n. 4). 


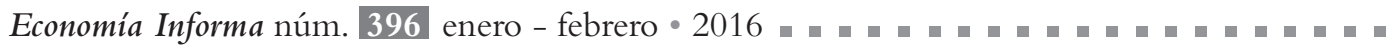

Siguiendo a Eduardo Gudynas, el extractivismo se refiere a la actividad económica que, bajo prácticas intensivas, remueve grandes volúmenes de recursos naturales que sin ser procesados (o serlo limitadamente) en su mayor parte son apropiados para ser exportados como alimentos o materias primas a los mercados globales (Gudynas, 2013: 1). En otras palabras, el extractivismo es la modalidad de crecimiento económico basada en la apropiación de la naturaleza, en un entramado productivo escasamente diversificado y dependiente de su inserción internacional como proveedor de materias primas y alimentos requeridos en las economías centrales.

El extractivismo es, en realidad, una ancestral modalidad de acumulación iniciada al momento de la colonización de lo que hoy es América Latina y la estructuración de la economía mundial bajo el orden colonial capitalista: ${ }^{2}$

Esta modalidad de acumulación extractivista estuvo determinada desde entonces por las demandas de los centros metropolitanos del capitalismo naciente. Unas regiones fueron especializadas en la extracción y producción de materias primas, es decir de bienes primarios, mientras que otras asumieron el papel de productoras de manufacturas. Las primeras exportan Naturaleza, las segundas la importan (Acosta, 2011: 85).

De hecho, uno de los rasgos característicos del desarrollo capitalista en América Latina, ha sido el extractivismo, aún más, "con América (Latina) el capitalismo se hace mundial, euro centrado y la colonialidad y la modernidad se instalan asociadas como los ejes constitutivos de su específico patrón de poder, hasta hoy" (Quijano, 2000: 342).

De esta manera, las causas de la persistencia del extractivismo en las economías latinoamericanas pueden encontrase en la importancia de ese patrón de acumulación adquirida desde la colonización europea, importancia que actualmente se refleja en el valor y volumen total de las exportaciones de los países de la región, al grado que en algunos de ellos las actividades extractivas son la mayor fuente de recursos del exterior ya sea para financiar las importa-

\footnotetext{
${ }^{2}$ De acuerdo con Alberto Acosta, la colonización y la colonialidad son categorías distintas: "Colonialidad es un concepto diferente de, aunque vinculado a, Colonialismo. Este último se refiere estrictamente a una estructura de dominación/explotación donde el control de la autoridad política, de los recursos de producción y del trabajo de una población determinada lo detenta otra de diferente identidad y cuyas sedes centrales están además en otra jurisdicción territorial. Pero no siempre, ni necesariamente, implica relaciones racistas de poder. El Colonialismo es obviamente más antiguo, en tanto que la Colonialidad ha probado ser, en los últimos 500 años, más profunda y duradera que el Colonialismo. Pero sin duda fue engendrada dentro de éste y, más aún, sin él no habría podido ser impuesta en la intersubjetividad del mundo de modo tan enraizado y prolongado" (Quijano, 2000, 342, n. 1).
} 
ciones o el gasto público, sin olvidar que en aquellos países donde prevalece la economía neoliberal son la fuente de renta más importante para el capital privado nacional y extranjero.

Las actividades extractivas, sin duda, generan diversos impactos sociales y ambientales cuya magnitud termina por limitar su posible aporte al desarrollo o a la construcción de una sociedad no capitalista, dado el elevado costo de los daños ambientales provocados por su persistencia en el conjunto de la economía:

En los mayores ingresos que supuestamente podrían percibir nuestras economías no se contabiliza la pérdida de biodiversidad, el deterioro de ecosistemas y de los servicios y funciones ambientales que prestan, la eventual desestructuración de culturas ancestrales, ni los recursos económicos que será necesario destinar para descontaminar el agua y la tierra. En la medida en que no se ha realizado un balance objetivo que de cuenta de los activos y pasivos que provocarán las nuevas explotaciones extractivas, la afirmación sobre mayores ingresos debe al menos relativizarse (Moncada, 2102: 2).

Estas críticas y muchas otras más, forman parte de las consideraciones imprescindibles al reflexionar y debatir sobre el rumbo que habrán de seguir nuestros países.

\section{Movimiento social y extractivismo}

El movimiento social que ha impulsado los cambios recientes en América Latina, se ha planteado la necesidad de una estrategia que permita superar el extractivismo depredador. A pesar de ello, se mantiene la modalidad extractivista a la que, con el calificativo de "progresista", parece atribuírsele una diferencia fundamental con la vieja modalidad extractivista que definió, fortaleció y profundizó la dependencia y hoy sostiene a la colonialidad.

Dicha diferencia radica en el hecho de que mientras los gobiernos neoliberales entregan los recursos naturales al capital, los gobiernos progresistas procuran una mayor legitimidad con el impulso a la redistribución de la renta de los hidrocarburos y los excedentes generados por las actividades extractivas para ampliar el gasto social, con lo que se mejoran las condiciones de existencia de la población.

Pero como el deterioro ambiental y la destrucción de la naturaleza no conocen de derechas y de izquierdas, la actividad extractiva provoca impactos ambientales negativos sin importar que las toleren o las emprendan gobiernos progresistas o reaccionarios. Aunque conviene decirlo, los gobiernos progre- 
sistas tienen como política explícita la recuperación de los recursos naturales en manos de empresas privadas y mantener un creciente respeto a la naturaleza, situación ajena a la actitud del capital.

El caso es que en Chile, los gobiernos de la Concertación que sucedieron a la dictadura, mantuvieron la estructura básica del sector minero y alentaron su ampliación a partir de estimular la inversión privada que, hoy, mantiene a los productos mineros, especialmente al cobre, como los principales productos de exportación que representaron $61 \%$ de las ventas totales chilenas al exterior (Gudynas, 2010: 13).

Por su parte, durante el gobierno de Lula da Silva en Brasil se convirtió en una potencia minera, política que ha continuado Dilma Rousseff. En efecto, en 2003, al comienzo de los gobiernos del PT, "la producción de cobre fue de 264 millones de toneladas y creció a 370 millones de toneladas en 2008" y en 2013 se triplico su producción. De esta manera, de acuerdo con datos proporcionados por la CEPAL, "las exportaciones provenientes de minas y canteras, que superaban los 6 mil millones de dólares en 2003, treparon a más de 21 mil millones de dólares en 2007" (Gudynas, 2010: 14).

Bajo los gobiernos progresistas de Lula y Dilma Rousseff, Brasil se ha convertido "en el más grande productor y exportador minero del continente. En este país se extrajeron más de 410 millones de toneladas de sus principales minerales en 2011, mientras que todas las demás naciones sudamericanas sumadas, se extrajeron poco más de 147 millones de toneladas" (Gudynas, 2013: 1). En este caso, los minerales principalmente exportados han sido el cobre, cinc, plomo, estaño, bauxita, carbón y hierro.

La explotación minera no está exenta de riesgos, los cuales aumentan con la minería a cielo abierto que implica la remoción de enormes cantidades de tierra por cada gramo de mineral extraído (en el caso del oro, la extracción de un kilogramo de este metal requiere remover 540 toneladas de materia), lo que se acompaña con el uso de procesos químicos altamente demandantes del agua: "Se calcula que, en el caso de la minería de metales, por cada tonelada de mineral crudo extraído se requieren entre 636 y 7123 litros de agua y que para los minerales no metálicos, este requerimiento fluctúa entre 136 y 4532 litros de agua por cada tonelada extraída" (Moncada, 2013: 4).

La utilización de elementos de alta nocividad, como el cianuro y el mercurio en la extracción mineral, llegan a contaminar en ocasiones de manera irreversible, los mantos freáticos y ocasionan graves daños a la salud humana y al ambiente que se manifiestan, principalmente, por el deterioro de la calidad 
de las aguas superficiales y subterráneas, el aire, los suelos, la vegetación y la fauna de los territorios donde se localiza la actividad minera.

La magnitud de la gravedad del daño, puede apreciarse si mencionamos lo que sucede en la región amazónica, ejemplo que se repite donde se asienta el extractivismo minero: "Más de un millón de personas en la región suramericana de la Amazonía padecen intoxicación por mercurio y otros residuos cancerígenos provenientes de la actividad minera, sin control y a expensas del ecosistema" (Mayoral, 2012: 21).

En Argentina, Brasil y Uruguay, se ha impulsado el "extractivismo agrícola", y con aliento incluso de los gobiernos progresistas, ha estado ocurriendo un cambio sustancial en la agricultura orientándola a monocultivos de exportación, sobre todo la soya: "Cultivo basado en variedades transgénicas, alto costo de maquinaria, herbicidas químicos, escaso o nulo procesamiento y exportación como commodity [...] Otro tanto sucede con los monocultivos forestales, que cubren amplias superficies y están volcados a elaborar pasta de celulosa" (Gudynas, 2010: 13).

El problema asociado al extractivismo agrícola, es la creciente utilización de territorio para producir commoditys con miras a la exportación, abandonándose la producción de alimentos para satisfacer las necesidades de la población; aún más, ese territorio, muchas veces es apropiado por empresa extranjeras legal o violentamente despojando a la población originaria o, a comunidades rurales. En un informe encargado por la Organización de las Naciones Unidas para la Agricultura y la Alimentación (FAO), publicado en mayo de 2009, se reconocía que: "El incremento de las compras masivas de terrenos en América Latina y otros continentes aumenta el riesgo de que los pobres se vean desposeídos o se les impida el acceso a la tierra y al agua", privatizadas a favor de empresas transnacionales. Así, en noviembre de 2007, "el conglomerado japonés Mitsui compró 100 mil hectáreas de tierras fértiles en Brasil -el equivalente a $2 \%$ de la superficie cultivada de Japón- para la producción de soya [demandada en el país asiático]. Las tierras están en Bahía, Minas Gerais y Maranhao" (Muresu, 2009: 20).

La magnitud de la producción de soya en Brasil, ha convertido a este país también en el líder del extractivismo agrícola:

Actualmente [Brasil] es el principal productor mundial de soya; en la zafra 2011-2012 superó los 66 millones de toneladas métricas (en nuestro continente le sigue Argentina con 40 millones de toneladas). Es también el primer exportador mundial y buena parte de lo que comercializa lo hace sin proce- 


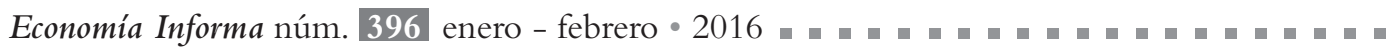

samiento. Este fenómeno va de la mano con un enorme aumento del área de cultivo, que ha superado los 24 millones de hectáreas (Gudynas, 2013: 3).

Lo significativo es que el impulso al extractivismo agrícola en Brasil, no sólo ha recibido la inyección de recursos del capital privado extranjero, sino también del gobierno. En los últimos tiempos el extractivismo agrícola:

Se ha beneficiado del más grande paquete de ayuda financiera estatal del continente (el llamado Plan Agrícola y Pecuario), que para los años 2011/2013, totalizó 115.2 miles de millones de reales destinados al crédito, lo que favorece directamente la expansión de la agroindustria exportadora en lugar de los pequeños agricultores (Gudynas, 2013: 4).

A su vez, en Argentina uno de los principales terrateniente del país resulta ser la empresa Benetton, propietaria de más de 900 mil hectáreas y, según la Federación Agraria de ese país, el 10\% del territorio argentino está en manos extranjeras. En algunas provincias la hectárea puede llegar a costar 8 dólares, lo que ha permitido a "empresas chilenas, europeas, norteamericanas y de países como Malasia, aprovechar el bajo precio del suelo para comprar amplias extensiones de terreno a lo largo del país" (Maresu, 2009: 30).

Es evidente que la estrategia de producción extractivista -los grandes proyectos de minería, monocultivo agroindustrial o la construcción de gigantescas obras de infraestructura-, termina por generar conflictos sociales y políticos de diversa intensidad con las comunidades afectadas enfrentando a los gobiernos con la población, situación que sin duda pone en riesgo la construcción de la sociedad posneoliberal.

En Ecuador, el presidente Rafael Correa al impulsar la minería a cielo abierto enfrentó la oposición de diversos grupos originarios y sociales a la Ley de Minería (aprobada por un Congreso provisional el 12 de enero de 2009). Los opositores a esa ley advertían que, al declararse a la actividad minera de "utilidad pública", se estaba autorizando la expropiación de tierras en territorios indígenas con sólo alegar un supuesto bienestar colectivo, contra lo cual los grupos originarios se encontraban indefensos pues si decidían defender sus territorios la ley los convertía en delincuentes.

Por supuesto, en el distanciamiento del gobierno de Correa con esa parte de la población, jugaron un papel de primordial importancia los medios de comunicación que en todo momento alentaron la creación de un clima de violencia y enfrentamiento que promovía la derecha y le permitía fortalecer sus anhelos golpistas. 
Actualmente, el área destinada en Ecuador para la explotación minera comprende 5.6 millones de hectáreas, equivalentes a $20 \%$ de la superficie total del país, incluidos parques nacionales y reservas naturales concesionadas desde la década de 1980 (Zibechi, 2009: 30).

De esta manera, el gobierno de Ecuador ha seguido impulsando, o por lo menos tolerando, la actividad extractiva que lo pone en el mismo rumbo de sus vecinos, particularmente de Perú donde los grupos indígenas opuestos a la explotación de petróleo en la selva y de la minería de cielo abierto en la región andina han sido violentamente reprimidos, lo cual ha enfrentado al gobierno de Ollanta Humalla con un amplio sector indígena.

Actualmente se calcula que 25 millones de hectáreas (casi una cuarta parte del país) están concesionadas a empresas mineras transnacionales que han hecho de Perú el primer productor de plata del mundo, tercero en estaño y zinc, cuarto de plomo y cobre, y quinto de oro. Y si bien los minerales representan $45 \%$ de las exportaciones, la actividad minera sólo aporta $4 \%$ de los ingresos del gobierno y ocupa 1\% de la población económicamente activa (Zibechi, 2009: 30).

En Argentina se sabe del funcionamiento de aproximadamente 15 nuevos yacimientos de oro, plata y cobre que afectan a 13 provincias, en especial la deforestación y la contaminación de los mantos acuíferos son algunos de los graves problemas, aunque no los únicos, que enfrenta la población. En este país, bajo el gobierno de Néstor Kirchner y los dos de Cristina Fernández, se siguió el mismo camino de impulsar la minería, particularmente en la zona andina, incluyendo un megaproyecto (Pascua Lama) compartido con Chile, que apunta a ser el segundo productor de oro continental (Gudynas, 2010: 14). Pero Ecuador, Perú o Argentina no son la excepción, casos similares se presentan en todos los países de la región.

En realidad a los directamente afectados por este tipo de actividades depredadoras, les sobran los motivos para oponerse, pues por un lado tienen un grave impacto sobre el medio ambiente y la población y, por el otro, porque aunque los contratos de explotación suscritos entre el gobierno y las empresas (casi todas extranjeras) incluyen medidas de seguridad, se carece de los instrumentos necesarios y adecuados para vigilar y sancionar los incumplimientos y las violaciones a lo convenido.

Pero hay algo más, en el afán de incrementar la inversión y mostrar cifras de crecimiento de la economía, muchos gobiernos ofrecen a las compañías extranjeras todas las ventajas posibles sin considerar el impacto en el ambiente y la salud de la población, dando un tratamiento más que generoso a la repa- 


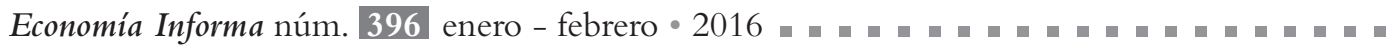

triación de ganancias, facilitando el pago mínimo de impuestos y regalías y permitiendo pagar salarios reducidos a los trabajadores que cumplen jornadas extenuantes.

De esta manera, la política que permite y alienta explotar sin medida a los trabajadores y recursos como el petróleo, el gas, los minerales, la selva o la tierra, para producir materias primas y alimentos en favor de la planta industrial y el consumo de los países centrales, sin considerar las necesidades de la población de las naciones dependientes, resulta para el país -no para su elite criolla- un muy mal negocio.

Privilegiar el crecimiento económico, ha significado mantener el extractivismo como el primer recurso, sobre todo si en el ámbito internacional existe una creciente demanda de productos primarios y alimentos, acompañada de una continua elevación de sus precios. Ciertamente, en el corto plazo se logra el crecimiento pero finalmente la economía se hace sumamente dependiente de exportaciones primarias para crecer.

\section{Consideraciones finales}

Bajo los gobiernos progresistas de América Latina, se está generando un patrón de acumulación basado en el "extractivismo progresista", donde el uso del excedente o la renta petrolera se orienta a mejorar las condiciones de vida sociales y a transformar la estructura productiva. Lo que establece una enorme diferencia con el extractivismo neoliberal.

El reconocimiento del esfuerzo que construye sociedades distintas a la neoliberal, no permite caer en la ilusión de creer que los gobiernos progresistas de la región han logrado dejar de depender del patrón extractivista para alcanzar los propósitos de una acumulación independiente y superar el neoliberalismo; pero sobre todo, no se puede decir que el cambio de finalidad política del sector extractivista haya permitido resolver los impactos sociales y ambientales que provoca la minería a cielo abierto o los monocultivos transgénicos.

De mantenerse en Latinoamérica la estrategia de sostener el crecimiento basado en las actividades de extracción, el libre comercio y la forma de integración actual en la economía mundial, a nuestros países les espera un futuro poco halagüeño. En el mejor de los casos, podrán mantener cierta dinámica con productos "refugio" como el oro, bastante apetecido por los especuladores internacionales para asegurar sus ganancias en momentos de crisis. Pero como muestra la experiencia reciente, el extractivismo puede arrojar datos positivos 
en el corto plazo, pero rápidamente muestra sus múltiples debilidades, tanto como los riesgos que supone para economías como las nuestras fincar sus posibilidades de futuro en un modelo como éste, ya fracasado tantas veces en el pasado cercano y lejano de América Latina.

\section{Referencias}

Acosta, Alberto (2011), "Extractivismo y neoextractivismo: dos caras de la misma maldición”, en Varios autores. Mas allá del desarrollo, Grupo Permanente de Trabajo sobre Alternativas al Desarrollo, Universidad Politécnica Salesiana/ Fundación Rosa Luxemburg, Quito, Ecuador, pp. 81-118.

Gudynas Eduardo (2013), "Brasil: el extractivista más grande del continente", Argenpress.info. Prensa argentina para todo el mundo, edición del 13 de mayo.

Gudynas, Eduardo (2011), "Debates sobre el desarrollo y sus alternativas en América Latina: una breve guía heterodoxa”, en Varios autores. Mas allá del desarrollo, Grupo Permanente de Trabajo sobre Alternativas al Desarrollo, Universidad Politécnica Salesiana/Fundación Rosa Luxemburg, Quito, Ecuador, pp. 21-53.

Gudynas, Eduardo (2010), "El nuevo extractivismo del siglo xxı”, Memoria. Revista de Política y Cultura, número 242, mayo, pp. 12-17.

Lander, Edgardo (2013), "Con el tiempo contado. Crisis civilizatoria, límites del planeta, asaltos a la democracia y pueblos en resistencia", en Grupo Permanente de Trabajo sobre Alternativas al Desarrollo Alternativas al capitalismo/colonialismo del siglo XXI, Fundación Rosa Luxemburg, Quito, Ecuador, pp. 27-62.

Marini, Ruy Mauro (1977/173), Dialéctica de la dependencia, México, ERA, Serie Popular, número $22,3^{\mathrm{a}}$ edición.

Mayoral, María Julia (2012), "Degradación en tierras amazónicas: conflictos en ascenso", en Varios autores, Abya Yala. Una visión indígena, Letras Urgentes, Prensa Latina, Agencia Informativa Latinoamericana, México.

Muresu, Stefanía (2009), "Privatización de tierras fértiles", Memoria. Revista de Política y Cultura, número 237, agosto-septiembre, pp. 18-20.

Moncada, Martha (2012), "Las falacias del neoextractivismo", en América Latina en movimiento. Agencia Latinoamericana de Información (ALAI), 7 de julio de 2012.

Quijano, Aníbal (2000), “Colonialidad del poder y clasificación social”, en Textos en homenaje a Immanuel Wallerstein, Journal World-Systems Research, Volumen XI, número 2, Verano/Otoño, pp. 342-386 (http;/jwsr.ucr.edu/).

Zibechi, Raúl (2009), "Ecuador: la lógica del desarrollo choca con los movimientos”, Memoria. Revista de Política y Cultura, número 237, agosto-septiembre, pp. 27-30. 